Supporting Information

\title{
Decontamination of mustard gas with processable dry reactive polymers via oxidation-chlorination
}

Yong-Chao Zheng, ${ }^{* a, c}$ Xue Geng, ${ }^{b}$ Sheng-Song Li, ${ }^{a, c} J i-N a W u,{ }^{a, c}$ Chao-Lin Xu, ${ }^{b} Y i$

Xin, ${ }^{a, c}$ Yan Cui, ${ }^{a, c}$ Chong-Lin Zhao, ${ }^{a, c}$ Lin Ye, ${ }^{* b}$ and Li-Kun Chen, ${ }^{* a, c}$

a. State Key Laboratory of NBC Protection for Civilian, Beijing, 102205, P. R. China.

b. School of Materials Science and Engineering, Beijing Institute of Technology, Beijing 100081,

P. R. China.

c. Research Institute of Chemical Defense, Beijing, 102205, P. R. China

E-mail address: zhengyongchao@mail.ipc.ac.cn; yelin@bit.edu.cn; clk930222@sohu.com 


\section{Contents}

1. The standard curve of HD concentration $\quad$ S1

2. NMR spectrum and GPC spectrum of PVM S2

3. Detoxification of $\mathrm{HD}$ by $\mathrm{PVMCl}$ with various monomer proportion $\mathrm{S} 3$

4. Copies of mass spectra of products $\quad$ S4-S5

5. Integration of the chromatographic peak areas S6

$\begin{array}{ll}\text { 6. Recyclability of PVMCl } & \text { S7 }\end{array}$

7. The miscibility of PVDF and PVM copolymer S8

8. SEM images of electrospun polymers S9

9. Comparison of the detoxification/degradation performance of solid S10 decontamination agents towards HD 
The standard curve of $\mathrm{HD}$ concentration

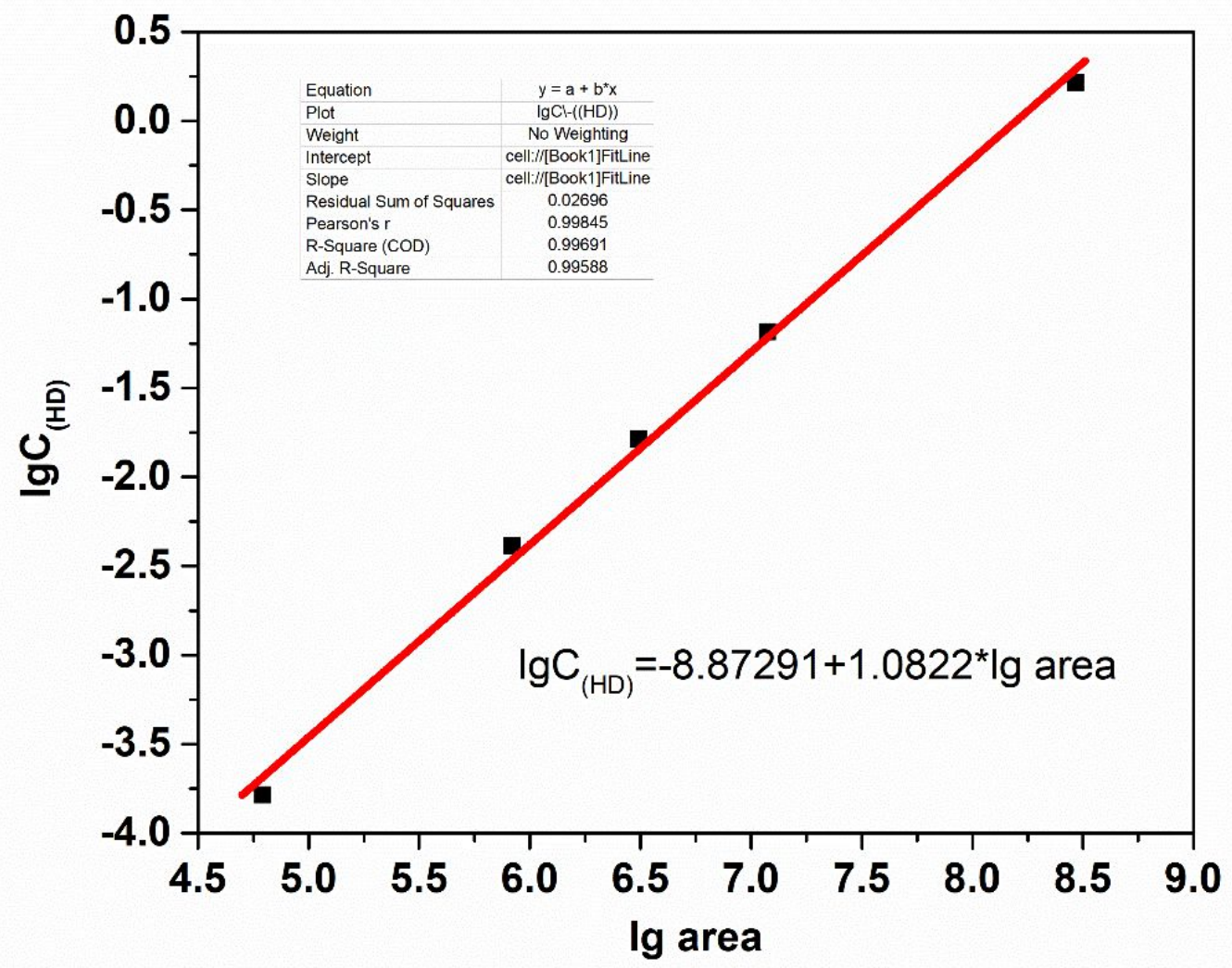

Figure S1. The standard curve of HD detoxification detected by Shimadzu GC2014C. 
NMR spectrum

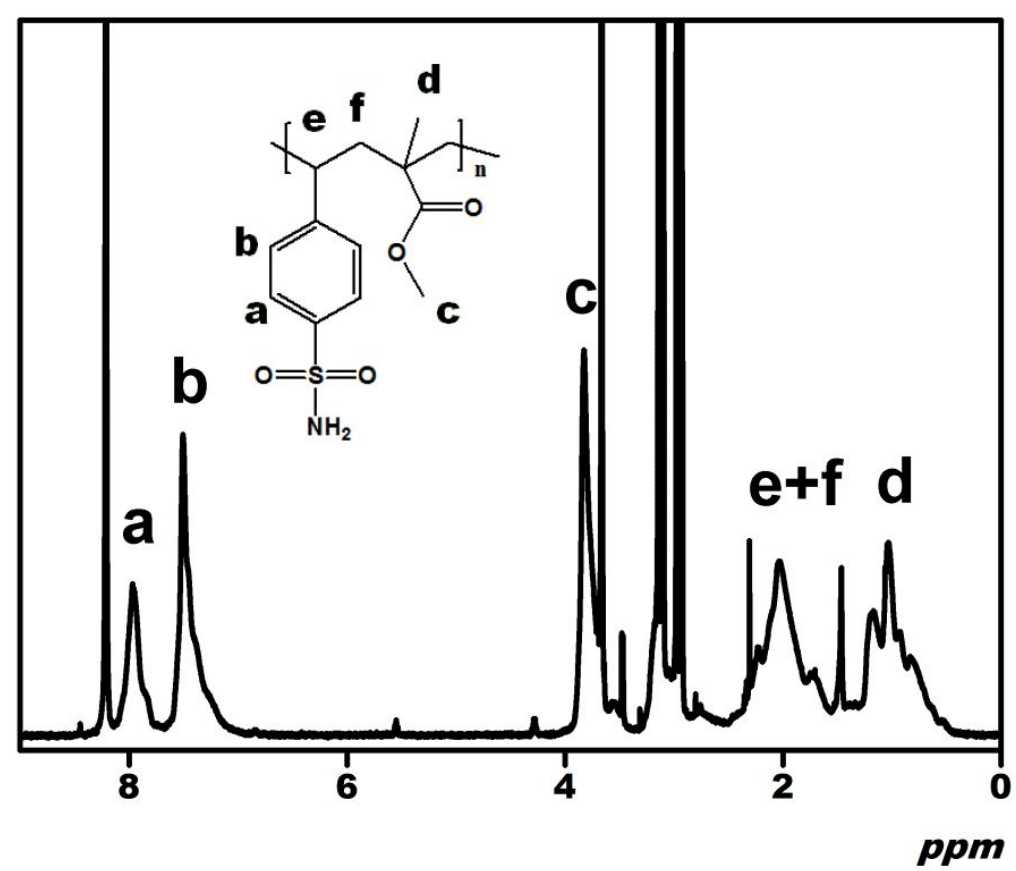

Figure S2. The NMR spectrum of PVM

GPC spectrum

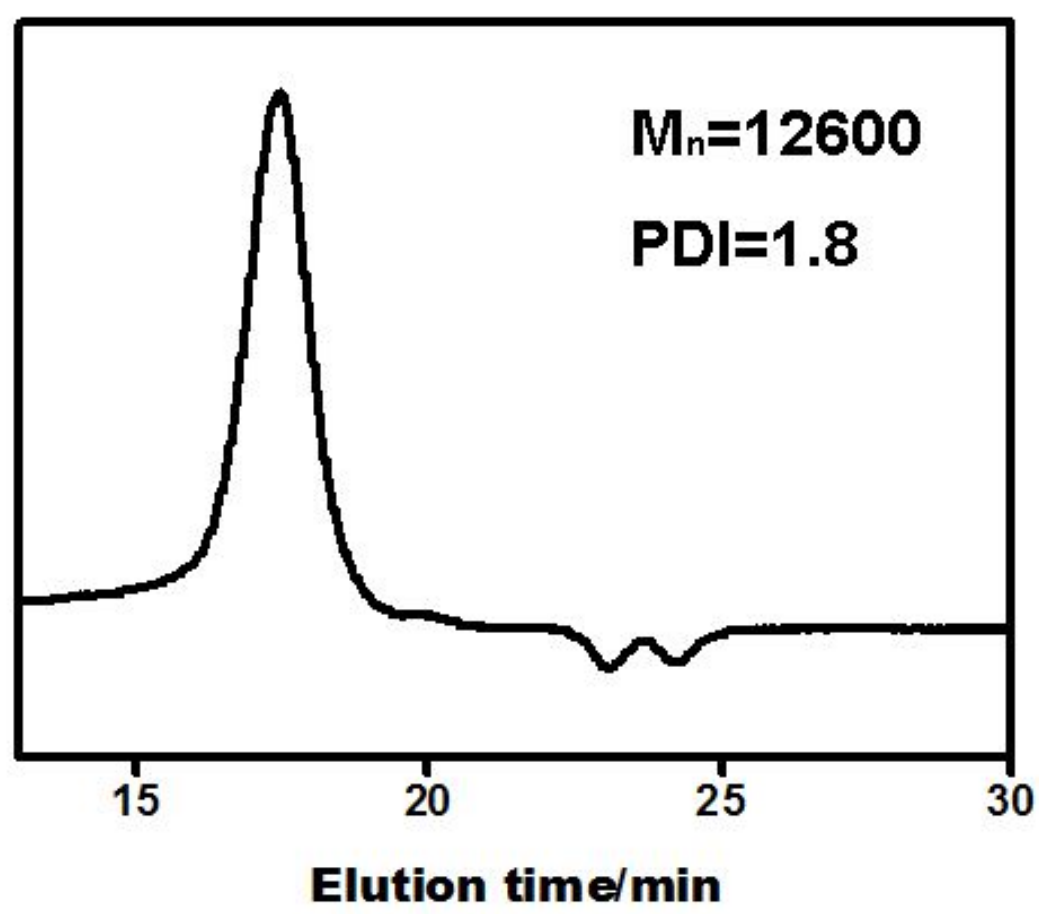

Figure S3. The GPC spectrum of PVM 
Detoxification of HD by copolymer with various monomer proportion

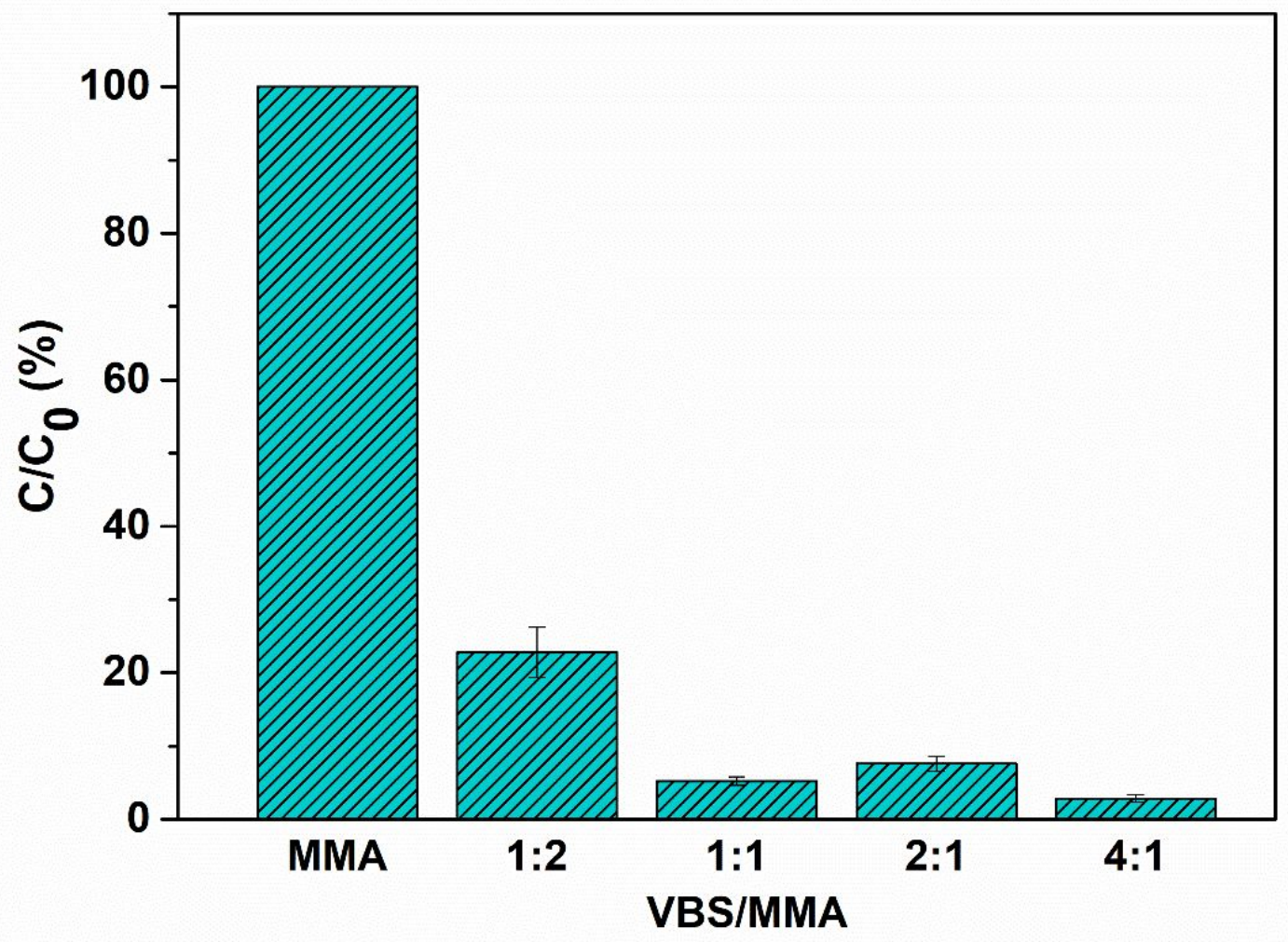

Figure S4. Detoxification rate of HD catalyzed by copolymer in $1 \mathrm{~h}$ with different ratio of VBS to MMA. 


\section{Copies of mass spectra of products}
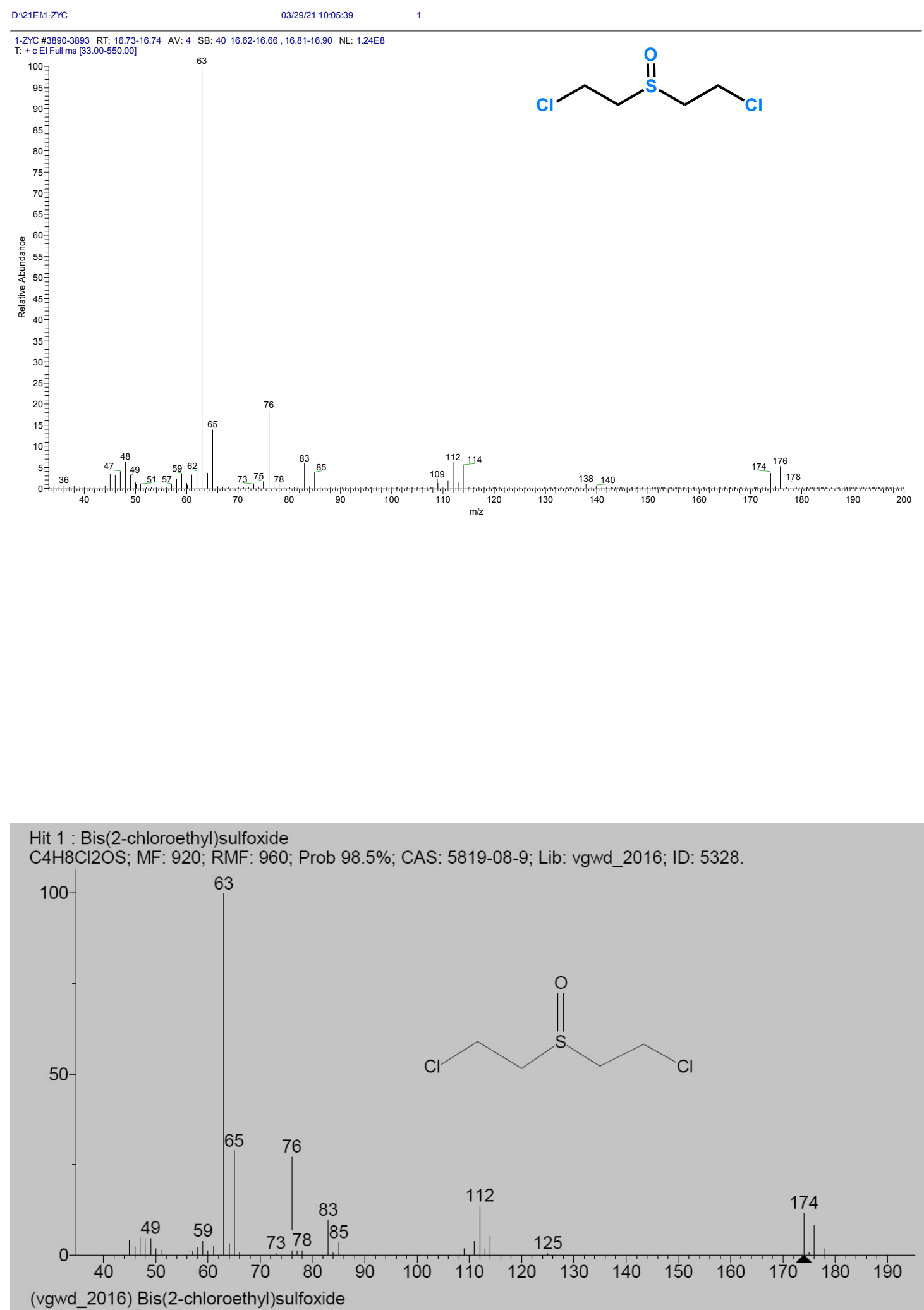
Figure S5. Mass spectrometry of product mustard sulfoxide detected by GC/MS (Agilent 7890A GC \& Agilent 5975C MSD) and the corresponding reference spectrum. (Match level:96\%)
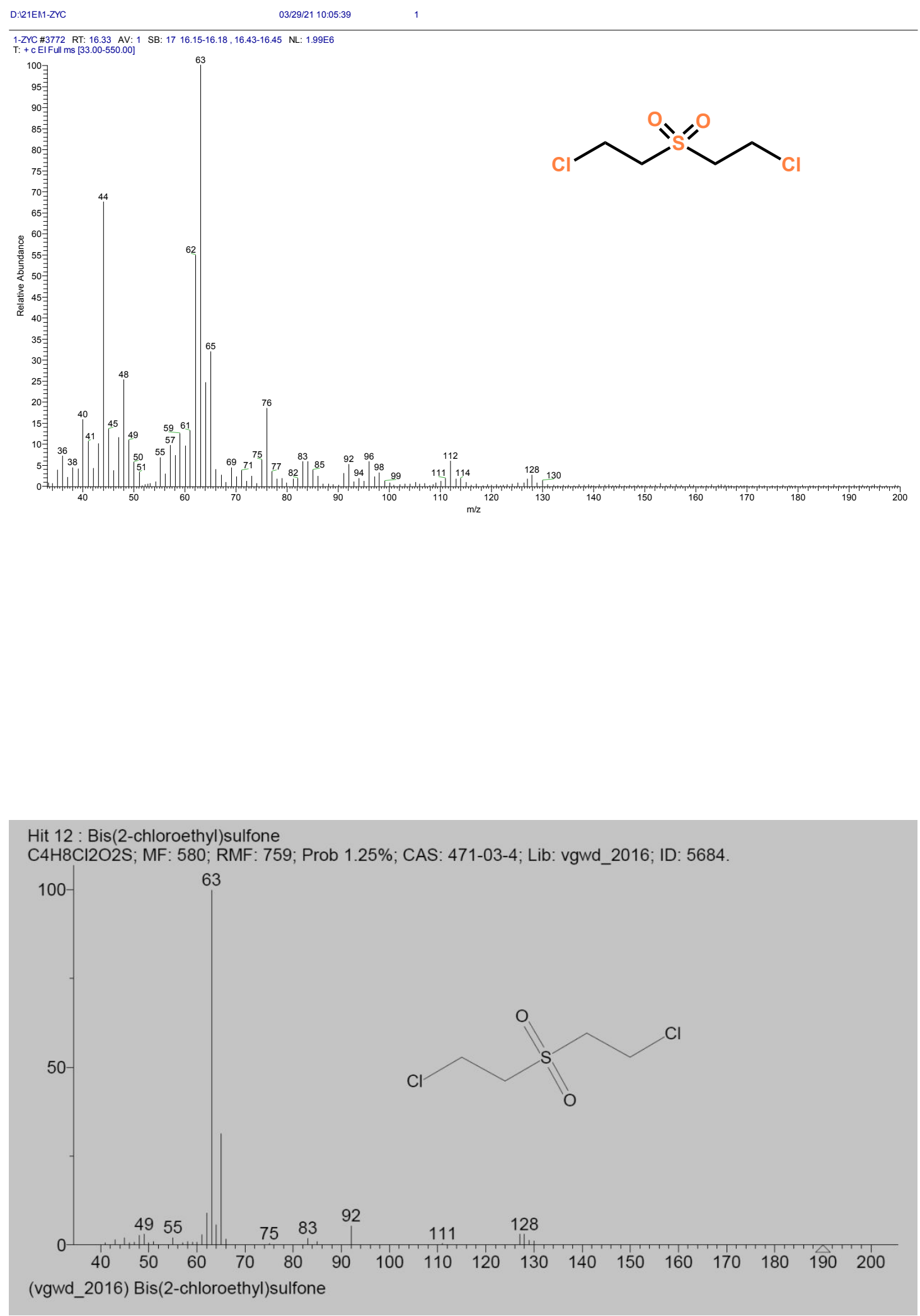
Figure S6. Mass spectrometry of product mustard sulfones detected by GC/MS (Agilent 7890A GC \& Agilent 5975C MSD) and the corresponding reference spectrum. (Match level:76\%) 
Integration of the chromatographic peak areas
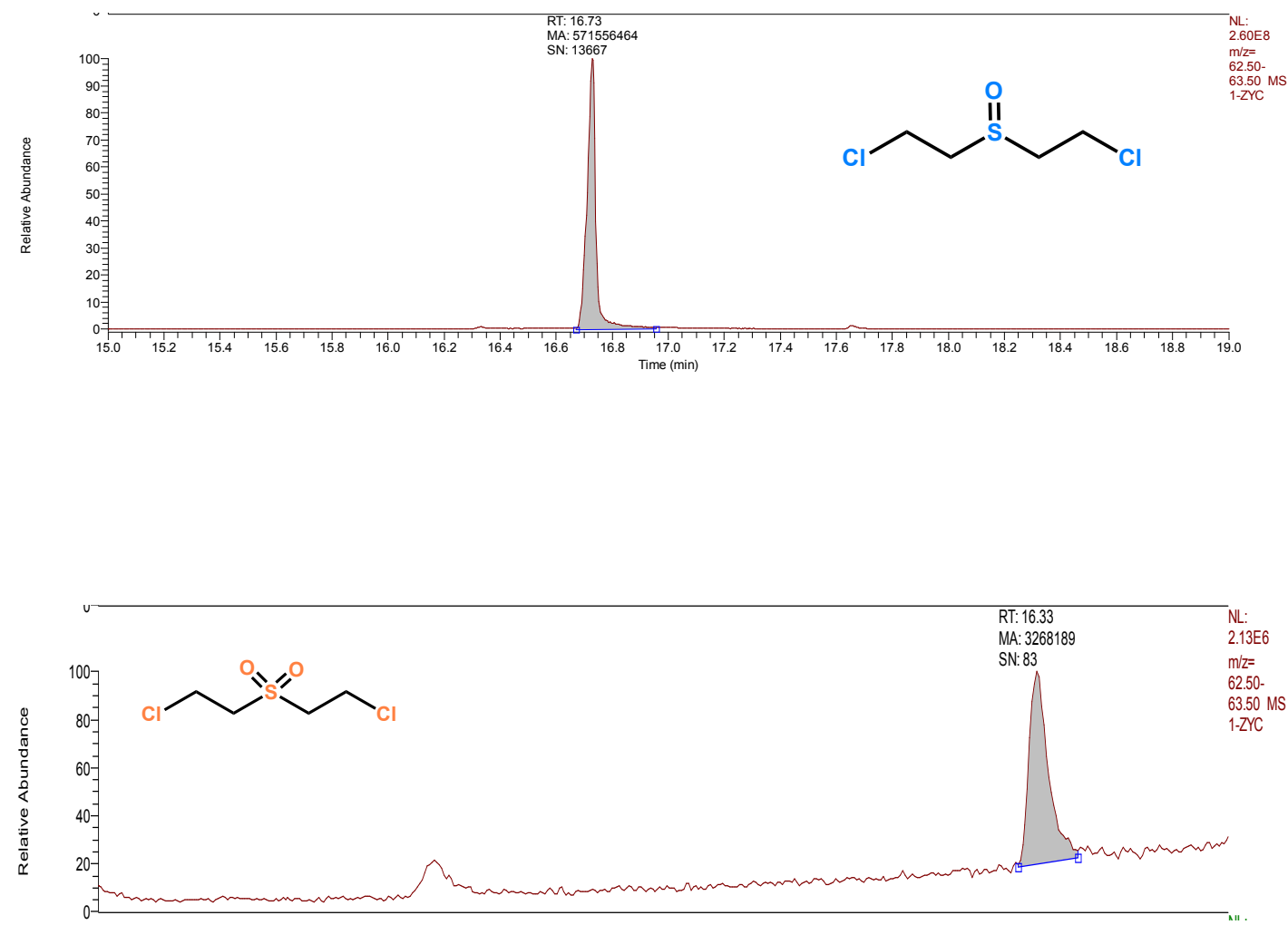

Figure S7. The comparison of chromatographic peak areas of mustard sulfoxide and mustard sulfones detected by GC/MS (Agilent 7890A GC \& Agilent 5975C MSD) and the corresponding reference spectrum. 


\section{Recyclability of PVMCl}

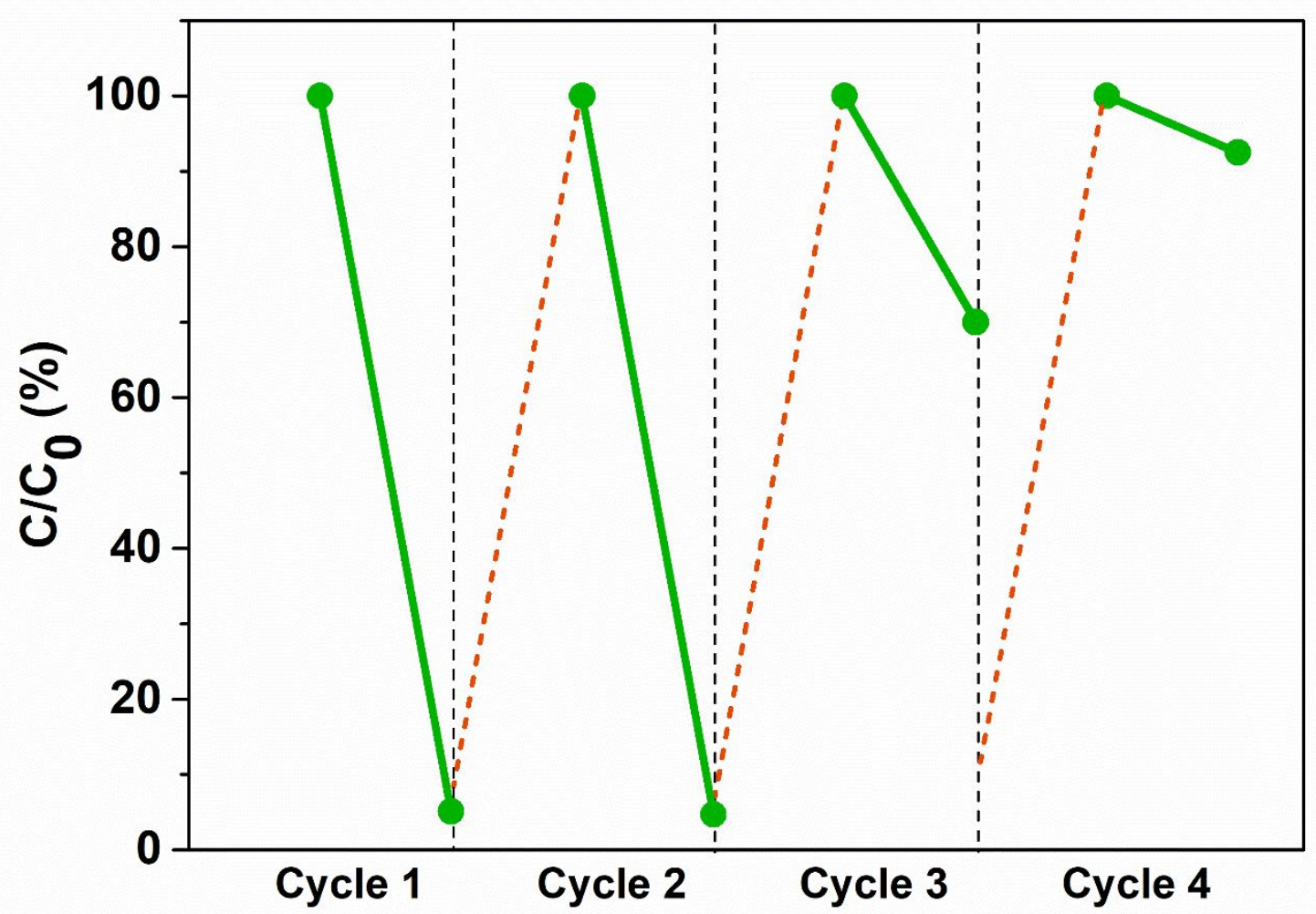

Figure S8. Recyclability of PVMCl for the detoxification of $H D$ 
The miscibility of PVDF and PVM copolymer

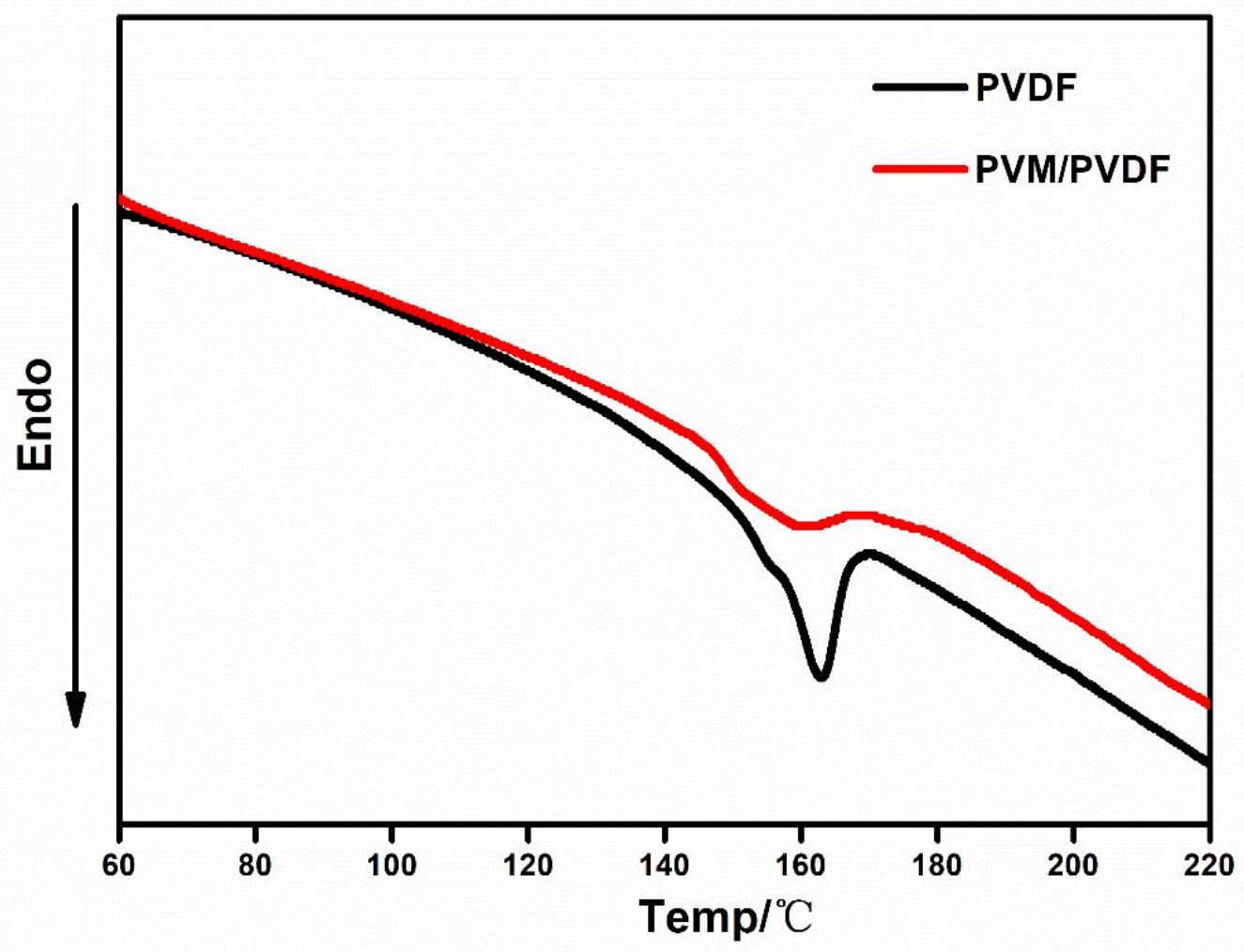

Figure S9. The DSC measurement of PVDF and PVM/PVDF copolymers 
SEM images of electrospun polymers
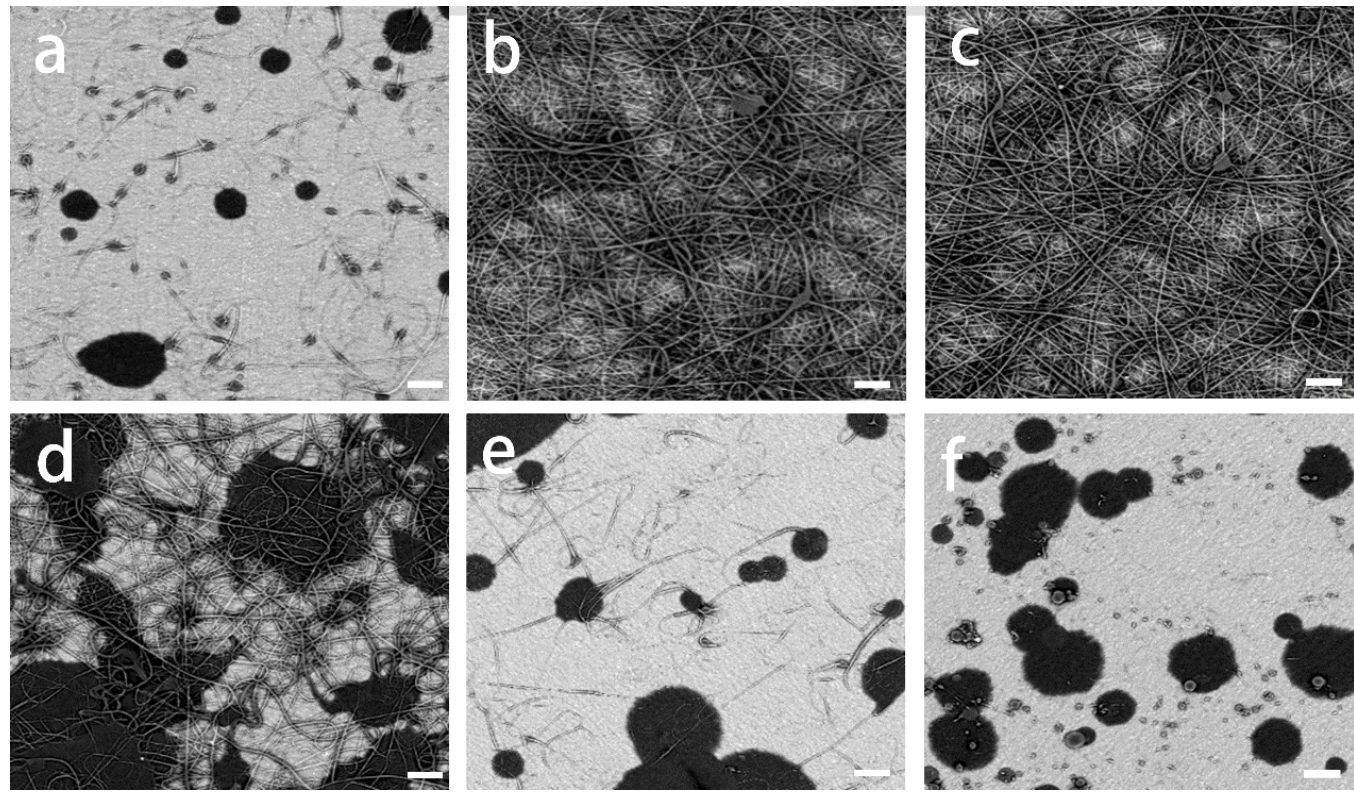

Figure S10. The SEM images of electrospun PVDF with concentration of (a) $0.11 \mathrm{~g} / \mathrm{mL}$,

(b) $0.14 \mathrm{~g} / \mathrm{mL}$, (c) $0.17 \mathrm{~g} / \mathrm{mL},($ d) $0.20 \mathrm{~g} / \mathrm{mL}$, (e) $0.23 \mathrm{~g} / \mathrm{mL}$, (c) $0.26 \mathrm{~g} / \mathrm{mL}$. scale bar: $20 \mu \mathrm{m}$.
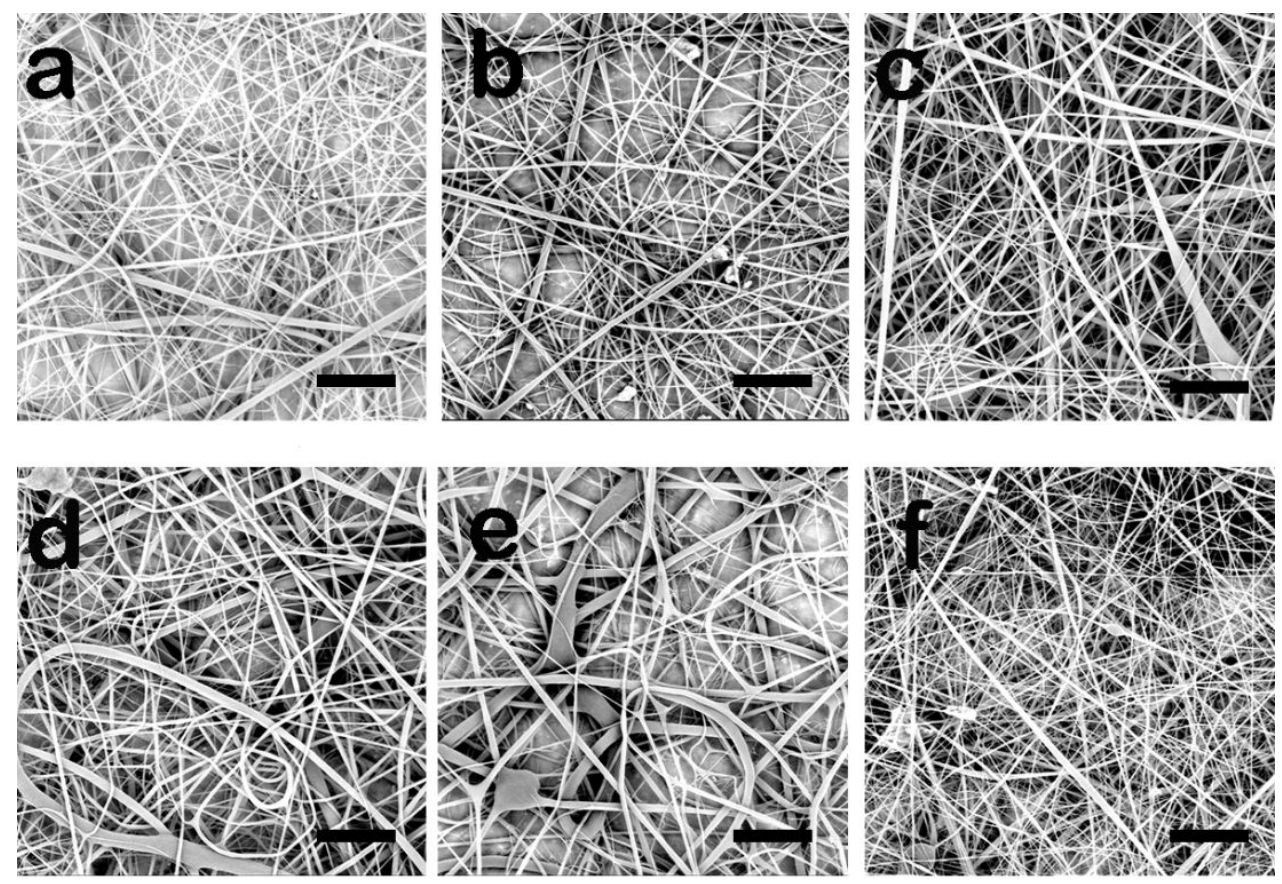

Figure S11. The SEM images of electrospun PVM/PVDF with mass ratio of (a) 3:17,

(b) 5:17, (c) 7:17, (d) 9:17, (e) 11:17, (c) 5:15. scale bar:10 $\mu \mathrm{m}$. 


\section{Comparison of the detoxification/degradation performance of solid decontamination agents towards HD}

Table S1. Comparison of the heterogeneous detoxification/ degradation performance towards HD or CEES on various solid decontamination agents

\begin{tabular}{|c|c|c|c|c|c|c|c|}
\hline Catalyst type & Additive & Substrate & $\begin{array}{l}\text { Reaction } \\
\text { temperature }\end{array}$ & $\begin{array}{c}\text { Reaction } \\
\text { time }\end{array}$ & $\begin{array}{c}\text { Mass ratio of } \\
\text { catalyst and sulfide }\end{array}$ & $\begin{array}{l}\text { Detoxification } \\
\text { rate }\end{array}$ & Ref. \\
\hline \multirow{3}{*}{ Polymers } & - & CEES & $25 \square$ & $2.5 \mathrm{~h}$ & $1: 1$ & $43.6 \%$ & $\begin{array}{c}\text { J. Mater. Sci. } \\
2009,44(8), 2069-2078\end{array}$ \\
\hline & - & CEES & $32 \square$ & $2 \mathrm{~h}$ & $5: 1$ & $87 \%$ & $\begin{array}{c}\text { Polymer } \\
138 \text { (2018) } 146-155\end{array}$ \\
\hline & $\mathrm{H}_{2} \mathrm{O}_{2}$ & CEES/HD & $20 \square$ & $1 \mathrm{~h}$ & $\begin{array}{c}\sim 2: 1 \\
\left(\mathrm{H}_{2} \mathrm{O}_{2}: \text { sulfide }\right)\end{array}$ & $100 \% / 95 \%$ & $\begin{array}{c}\text { ACS Appl. Polym. Mater. } \\
2020,2,4640-4646\end{array}$ \\
\hline \multirow{4}{*}{$\begin{array}{l}\text { Nanocrystalline } \\
\text { metal oxides }\end{array}$} & - & HD & $30 \square$ & $24 \mathrm{~h}$ & $250: 1$ & $\sim 95 \%$ & $\begin{array}{l}\text { J. Hazard. Mater. } \\
167 \text { (2009) } 1192-1197\end{array}$ \\
\hline & - & HD & $25 \square$ & $64 \min$ & $50: 1$ & $95 \%$ & $\begin{array}{c}\text { Mater. Res. Bull. } \\
46 \text { (2011) 2050-2056 }\end{array}$ \\
\hline & - & HD & $25 \square$ & $64 \mathrm{~min}$ & $50: 1$ & $95-98 \%$ & $\begin{array}{c}\text { J. Hazard. Mater. } \\
192 \text { (2011) 1491- } 1504\end{array}$ \\
\hline & - & HD & $25 \square$ & $64 \min$ & $50: 1$ & $95 \%$ & $\begin{array}{c}\text { J. Hazard. Mater. } \\
227-228 \text { (2012) 62- } 67\end{array}$ \\
\hline \multirow{2}{*}{$\begin{array}{l}\text { Activated } \\
\text { Carbon }\end{array}$} & $\mathrm{H}_{2} \mathrm{O}$ & HD & $160 \square$ & $0.5 \mathrm{~h}$ & $160: 1$ & $100 \%$ & $\begin{array}{c}\text { Carbon } \\
49 \text { (2011) 3899-3906 }\end{array}$ \\
\hline & $\mathrm{H}_{2} \mathrm{O}_{2}$ & HD & $25 \square$ & $3 \mathrm{~h}$ & $20: 1$ & $90-95 \%$ & $\begin{array}{c}\text { Environ. Sci. Technol. } \\
2014,48,10912-10918\end{array}$ \\
\hline PVMCI & & HD & $25 \square$ & $1 \mathrm{~h}$ & $8: 1$ & $94.7 \%$ & This work \\
\hline
\end{tabular}

